Aims. Early interventions are recommended in adolescents to prevent long-term psychiatric morbidity. However, in Low and Middle Income Countries (LMICs), where there are no child and adolescent mental health services, early identification of adolescents at-risk of mental health problems remains a challenge. Pediatric Symptoms Checklist (PSC) is used in preventive child healthcare services in a number of high income countries for early identification of children and adolescents in need of mental health services. The aim of this study was to assess the reliability and validity of self-rated, Urdu version of PSC to identify at-risk adolescents studying in the public schools of rural Rawalpindi in Pakistan.

Method. We did a cross-sectional epidemiological survey with all adolescents aged 13-15 years, studying in 41 public schools of Kallar Syedan sub-district in Rawalpindi, Pakistan. An adapted Urdu version of self-reported PSC was used to assess the psychosocial distress in adolescents in-terms of externalizing, internalizing and attention problems. Strengths and Difficulties Questionnaire (SDQ) was used as a gold standard measure. Youth version of PSC and SDQ were administered in classroom settings by trained research teams.

Result. The data were collected from 5856 adolescents (response rate 97\%) between April-May, 2019. The mean age of the participants was 14.37 years $( \pm 1.06) ; 51 \%$ participants were female. The internal consistency reliability of Urdu version of PSC was good (Cronbach alpha 0.85). At the standard cut-off score of PSC $\geq 28$, the prevalence rate of psychosocial distress in adolescents was $25.5 \%$ ( $27.4 \%$ in boys \& $23.6 \%$ in girls). Using the SDQ total difficulties score $\geq 16$ as a standard criterion; the area under the ROC curve was 0.85 (95\% CI 0.82-0.88), with a sensitivity of $57.64 \%$ and specificity of $89.10 \%$ of PSC. If the sensitivity and specificity of PSC is optimized to $76 \%$ at the cut-off score of PSC $\geq 24$, the prevalence rates of psychosocial distress in adolescents is increased to $41 \%$.

Conclusion. In our study, 1 in 4 adolescents in public schools of rural Rawalpindi in Pakistan have been identified at-risk of poor socio-emotional development. Urdu version of PSC is a reliable and valid tool to identify adolescents in need of psychosocial interventions in public schools of rural Pakistan. While the standard cut-off score yields a better specificity; PSC with relatively lower cutoff score can be used a screening tool to identify at-risk adolescents in public schools of rural Pakistan.

\section{A UK-wide survey of Balint, support groups and psychotherapy training opportunities for SAS (Specialty Doctors and Associate Specialists) Psychiatrists}

Alina Vaida* and Masud Awal

Birmingham and Solihull Mental Health NHS Foundation Trust ${ }^{*}$ Corresponding author.

doi: 10.1192/bjo.2021.197

Aims. To investigate SAS Psychiatrists' opportunities for Balint-type, support groups and psychotherapy training opportunities nationwide, for which there is a lack of existing literature or established framework.

Method. An online questionnaire was sent to UK-wide SAS psychiatry doctors with the support of the RCPsych Speciality Doctors and Associate Specialist Psychiatrists Committee (SASC). The survey enquired about location, work experience, future plans, Balint-type groups, psychotherapy opportunities and support.

Result. 122 doctors completed the questionnaire, estimated to constitute approximately $8 \%$ of SAS psychiatry posts (or more if considering all vacancies), based on the RCPsych Census (2015), from across all UK nations. Time spent in an SAS role varied widely between months (10\%) to over 20 years (5\%), with the median and mode being $8-12$ years (25\%). Regarding future career plans $61 \%$ responded that they would be considering either the Certificate of Eligibility for Specialist Registration (CESR) route, or applying for future training or both.

$24 \%$ reported being part of a Balint-type group whilst almost double this number (47\%) said they would be interested to join but none were available. $31 \%$ were part of a reflective practice or support group whilst $44 \%$ reported that they were interested in joining but none were available. Only $7 \%$ said that they were not participating or not interested in either a Balint group or a reflective group. Free-response comments suggested these opportunities were usually reserved for trainees and service commitments prevented attendance.

$76 \%$ of respondents reported access to an SAS Tutor, but only $21 \%$ confirmed access to a psychotherapy tutor.

Half of respondents indicated they did not have access to information and guidance they needed regarding accessing psychotherapy opportunities, with only $27 \%$ thinking they did.

$24 \%$ reported managing to gain experience in at least one psychotherapeutic modality, $44 \%$ of whom received medical psychotherapist supervision; whilst $13 \%$ said they did not intend to pursue this.

Conclusion. The results highlight that interest in joining Balint and reflective support groups significantly exceeds local provision. As these groups are not mandatory requirements for CESR application, the interest expressed (including amongst those reporting to be SAS by choice) suggests that SAS Psychiatrists value these opportunities for their recognised professional developmental and clinical benefits; these include peer support, understanding doctor-patient interactions and having a space to reflect on the emotional impact of clinical work. Trusts should consider supporting SAS doctors wishing to join new or existing Balint-type or other supportive reflective clinician groups.

\section{Sociodemographic, clinical and personal}

characteristics of patients with borderline personality disorder in a public general hospital in Lima, Peru during the first wave of the COVID-19 pandemic

\section{Glauco Valdivieso}

Instituto Peruano para el Estudio y Abordaje Integral de la Personalidad, Servicio de Psiquiatría, Hospital de Emergencias Villa EL Salvador

\section{doi: 10.1192/bjo.2021.198}

Aims. To describe the main characteristics of adolescent and adult patients with Borderline Personality Disorder (BPD) treated in Emergency and Hospitalization services of Villa El Salvador Emergency Hospital during the first wave of the COVID-19 pandemic in Lima, Peru.

Method. An analysis of 17 cases of patients with BPD according to DSM 5 criteria was carried out in SISGALEN PLUS software database that have been evaluated in the Emergency and Hospitalization areas during the first wave of the COVID-19 pandemic. Sociodemographic, clinical and personal variables were taken into account. A descriptive analysis of frequencies and proportions was carried out in SPSS 24.0 software.

Result. Regarding sociodemographic variables, the average age was 27.47 ( $\mathrm{SD}=11.242$ ), $82.4 \%$ single, $88.2 \%$ female, $52.9 \%$ from Villa El Salvador, $82.4 \%$ catholics, $76.5 \%$ have completed secondary school and $47.1 \%$ were housewives. For clinical variables, $64.7 \%$ 